\title{
Updated Powder Diffraction notes for authors
}

The Powder Diffraction (PD) journal receives about 100 papers each year. Many of these submitted papers, especially those prepared by new authors, have mechanical errors and needed to be corrected before they can be accepted for publication. This correction process can have a significant effect on the total time of editing, acceptance, and publication of a paper. Therefore, the PD notes for authors have recently been updated to state clearly its paper requirements. The updated version of the notes is published in this issue and also posted on the PDJ page of the ICDD website (http://www.icdd.com/ resources/pdj). PD authors should check and make sure that their papers are prepared properly before submitting to the PD Peers-X-Press (PXP) system, the online paper and peer review system for this journal hosted by the American Institute of Physics.

To bring to the attention of PD authors, some of the main requirements for preparing a paper are also briefly described in this editorial.

Section headings are used to divide a large body of information reported in a paper into logical chucks. Most of the submitted papers use single-level headings, but a maximum of four heading levels can be used in PD. For example, it is useful to use level-two headings in the Results and Discussion Section to report and discuss the results obtained by different experimental and data-analysis techniques. The format for levels of section headings is given in Fig. 1 of the updated notes for authors.

Italics are used to denote text in non-English languages and variables in equations and to distinguish between elements and nonelements. For examples, ab initio, et al., in situ, etc., should be in italics. More style guidelines for PD are given in the updated notes for authors.

The author-date referencing system, not the numeric system, is used in PD for citations in the body of the main text and the bibliography (or reference list) at the end of the text. A citation in the reference list should include correct spelling of all author names, year of publication, article title, and the page numbers. The inclusion of an article title helps readers to understand the content of the citation before deciding whether or not to retrieve the reference. It should also be noted that the citations in the reference list must be given in the alphabetical order of the family names of the first authors. The examples for properly formatted citations of journal article, book, article in book, computer program, etc., are given in the updated notes for authors. Please note that punctuation and proper format used in a PD citation can be different from those of other journals.

PD encourages authors to report powder diffraction data for a compound in a PD paper. The data should report in a diffraction-data table, and the contents of the table must include both observed and calculated values of $2 \theta, d$, and $I$. The observed values should be determined directly from the experimental pattern without using unit-cell information on the corresponding compound. The calculated values are calculated from the refined unit-cell parameters and the Miller indices $(h k l)$ obtained from either the automatic indexing or the unit-cell parameter refinement process. The calculated values of $I$ can be omitted if they are unavailable. The required data columns and more information on reporting diffraction data are given in the updated notes for authors.

Tables and figures should not be imbedded within the running text of a paper. Each table is listed in separate page after the reference list, while each figure without figure caption is uploaded electronically to PXP as noted in the submission guide of PXP. The figure captions of all figures are listed in a separate page after the table pages at the end of the paper.

I hope that the authors will find the updated notes for authors as a useful guide for preparing and submitting papers for possible publication in PD. If you have any other editorial questions, please contact me at tinghuang@tinghuang.com or the managing editor at boris@icdd.com.

Ting C. Huang Editor-in-Chief 